Review Article

\title{
Dental Tissue-Derived Human Mesenchymal Stem Cells and Their Potential in Therapeutic Application
}

\author{
Lu Gan, ${ }^{1}$ Ying Liu, ${ }^{1}$ Dixin Cui, ${ }^{1}$ Yue Pan, ${ }^{1}$ Liwei Zheng, ${ }^{1}$ and Mian Wan $\mathbb{D}^{2}$ \\ ${ }^{1}$ State Key Laboratory of Oral Diseases and National Clinical Research Center for Oral Diseases and Department of \\ Pediatric Dentistry, West China Hospital of Stomatology, Sichuan University, Chengdu, Sichuan 610041, China \\ ${ }^{2}$ State Key Laboratory of Oral Diseases and National Clinical Research Center for Oral Diseases and Department of Cariology \\ and Endodontics, West China Hospital of Stomatology, Sichuan University, Chengdu, Sichuan 610041, China
}

Correspondence should be addressed to Mian Wan; mianwan@scu.edu.cn

Received 26 June 2020; Accepted 15 July 2020; Published 1 September 2020

Academic Editor: Sangho Roh

Copyright @ $2020 \mathrm{Lu}$ Gan et al. This is an open access article distributed under the Creative Commons Attribution License, which permits unrestricted use, distribution, and reproduction in any medium, provided the original work is properly cited.

\begin{abstract}
Human mesenchymal stem cells (hMSCs) are multipotent cells, which exhibit plastic adherence, express specific cell surface marker spectrum, and have multi-lineage differentiation potential. These cells can be obtained from multiple tissues. Dental tissue-derived hMSCs (dental MSCs) possess the ability to give rise to mesodermal lineage (osteocytes, adipocytes, and chondrocytes), ectodermal lineage (neurocytes), and endodermal lineages (hepatocytes). Dental MSCs were first isolated from dental pulp of the extracted third molar and till now they have been purified from various dental tissues, including pulp tissue of permanent teeth and exfoliated deciduous teeth, apical papilla, periodontal ligament, gingiva, dental follicle, tooth germ, and alveolar bone. Dental MSCs are not only easily accessible but are also expandable in vitro with relative genomic stability for a long period of time. Moreover, dental MSCs have exhibited immunomodulatory properties by secreting cytokines. Easy accessibility, multi-lineage differentiation potential, and immunomodulatory effects make dental MSCs distinct from the other hMSCs and an effective tool in stem cell-based therapy. Several preclinical studies and clinical trials have been performed using dental MSCs in the treatment of multiple ailments, ranging from dental diseases to nondental diseases. The present review has summarized dental MSC sources, multi-lineage differentiation capacities, immunomodulatory features, its potential in the treatment of diseases, and its application in both preclinical studies and clinical trials. The regenerative therapeutic strategies in dental medicine have also been discussed.
\end{abstract}

\section{Introduction}

Human mesenchymal stem cells (hMSCs) are multipotent cells isolated from various tissues, including bone marrow, adipose tissue, umbilical cord, and dental tissue. These cells share similar properties: being plastic-adherent, expressing a specific cell surface marker spectrum (CD73+, CD90+, CD105+, CD34-, CD45-, CD11b-, CD14-, CD19-, CD79a-, and human leucocyte antigen-DR-), and possessing the ability to give rise to osteoblasts, chondrocytes, and adipocytes. hMSCs are highly accessible and expandable in vitro with genomic stability. Furthermore, these cells have the remarkable potential of multipotent differentiation, as they not only could differentiate into mesodermal lineages (adipocytes, osteocytes, and chondrocytes) but also could transdifferentiate into ectodermal lineages (neurocytes) and endodermal lineages (hepatocytes and pancreocytes). All these characteristics make them promising stem cell sources for regenerative therapy, but their clinical applications have been limited due to questionable safety issues, inconclusive quality control, unaccomplished clinical-grade production, and incomplete understanding of the mechanism regulating these hMSCs [1-4].

To address this, scientists worldwide have been searching for safe, effective, and easily accessible stem cell sources with great differentiation potential for regenerative medicine. Dental MSCs, which show typical MSC features, have been found in various dental tissues, ranging from discarded extracted teeth to their attached tissues [5-7]. These cells are not only easily accessible but are also expandable with relative genomic stability for a long period of time. Notably, 


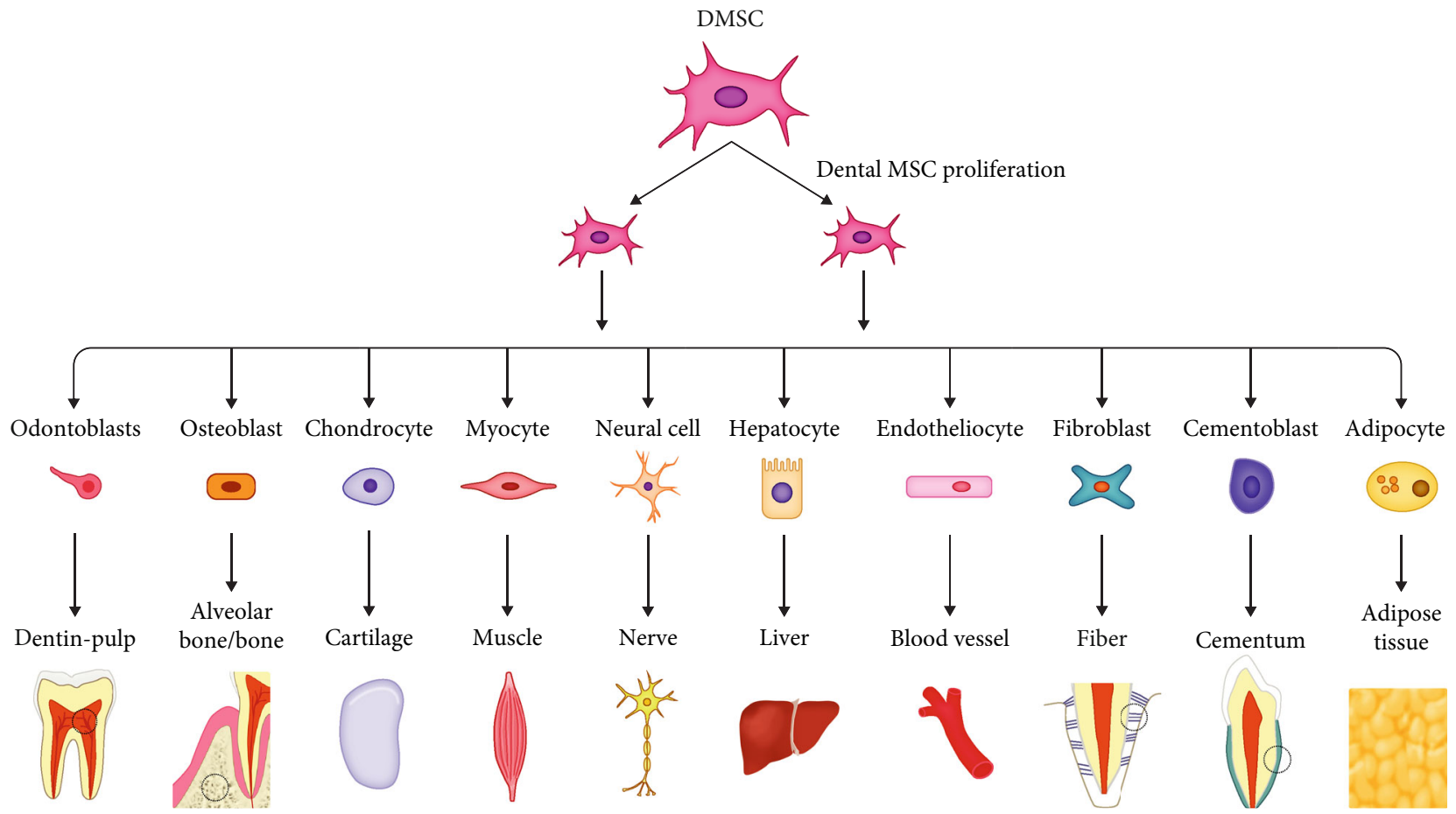

FIgURE 1: Multilineage differentiation capacity of dental MSCs.

apart from mesodermal lineages, they have the ability to transdifferentiate into ectodermal and endodermal lineages [5, 8-12] (Figure 1). Moreover, dental MSCs exhibit immunomodulatory properties by secreting cytokines and immune receptors [13]. All these characteristics of dental MSCs make them distinct from other hMSCs, and they can be applied in stem cell-based therapy. Several preclinical studies and clinical trials were performed using dental MSCs in the treatment of dental diseases and nondental diseases like neurodegenerative diseases and autoimmune and orthopedic disorders [14-18].

The present review has summarized dental MSC sources, multi-lineage differentiation potential, immunomodulatory features, its potential in the treatment of diseases, and its application in both preclinical studies and clinical trials. The regenerative therapeutic strategies in dental medicine have also been discussed.

\section{Dental Mesenchymal Stem Cells (Dental MSCs)}

The existence of dental MSCs was suggested by the formation of tertiary dentin following dental caries or trauma. The first efficient population of dental MSCs was reported from the dental pulp tissue of an extracted third molar [19]. Later on, cells that possess characteristics of MSCs were isolated from the pulp tissue of exfoliated deciduous teeth [20], apical papilla [21], periodontal ligament [22], gingiva [23], dental follicle [24, 25], tooth germ [26], and alveolar bone [27]. These cell populations exhibit heterogeneity, i.e., distinct cell surface markers, proliferation rate, and differentiation poten- tial, which has been reviewed by Zhou et al. [28], suggesting their diverse functions and applications in clinic.

2.1. Stem Cells from Dental Pulp. The first dental MSC population, human dental pulp stem cells (hDPSCs), was isolated from the dental pulp tissue of impacted third molars. These cells exhibit MSC properties, including high proliferation, multi-lineage differentiation potential, as well as immunomodulatory properties $[19,29]$. Substantial studies have documented the odontogenic differentiation potential of hDPSCs, i.e., hDPSCs generated a dentin-pulp-like organoid with Matrigel in vitro and induced mineralized reparative dentin formation with hydroxyapatite (HA)/tricalcium phosphate (TCP) ceramic particles in vivo [19, 30-32]. Attributing to the origin of the neural crest, hDPSCs show remarkable neurogenic potential compared with human bone marrow stem cells (BMMSCs). The higher expression level of neurotrophins like nerve growth factor (NGF) and longer axons were detected in hDPSCs cultured with a microfluidic coculture system containing trigeminal neurons. Neurospheres were also generated by hDPSCs upon specific differentiation conditions [33-35]. The ability of hDPSCs to differentiate into endothelial cells and their angiogenic potential have also been reported, as hDPSCs were found to secrete vascular endothelial growth factors (VEGF) and generate visible blood vessels in three-dimensional- (3D-) printed HA constructs [36]. Their capabilities of neurogenic and angiogenic differentiation made a great contribution to the whole pulp regeneration. Implanted hDPSCs gave rise to $3 \mathrm{D}$ pulp tissue with vascular and nerve reconstruction in the empty root canal of traumatized permanent incisors [37]. hDPSCs could also differentiate into osteoblasts and 
further regenerate bone tissue, due to expressing several typical osteoblastic markers, such as alkaline phosphatase (ALP), osteopontin (OPN), and osteocalcin (OCN) [38]. Newly formed bone was found following the application of the bioengineered constructs of hDPSCs with poly- $\varepsilon$-caprolactone- (PCL-) biphasic calcium phosphate (BCP) scaffolds. hDPSCs could also differentiate into other cell lineages, such as adipocytes, chondroblasts, hepatocytes, and cardiomyocytes. The high plasticity of hDPSCs makes them an ideal stem cell source for stem cell-based therapy, which has been thoroughly reviewed by Mortada et al. [29].

Then, stem cells from the dental pulp tissue of exfoliated deciduous teeth were purified with a similar method for hDPSC isolation. Analogous to hDPSCs, cultured stem cells from exfoliated deciduous teeth (SHEDs) are capable of differentiating into various cell types, such as osteocytes, chondrocytes, adipocytes, odontoblasts, endothelial cells, and hepatocytes [20]. However, due to the developmental differences between deciduous and permanent teeth, SHEDs present different features from hDPSCs, for instance, a higher proliferative capability, more cell-population doublings, a sphere-like cluster formation, and a distinctive osteoinductive capacity [20]. For odontogenic differentiation potential, SHEDs are able to differentiate into odontoblasts and form dentin-like tissue or pulp-like tissue, instead of a complete dentin-pulp-like complex [20,39]. When combined with collagen type I, hDPSCs formed the functional dental pulp tissue in the full-length root canal. The newly formed pulp tissue contained functional odontoblasts, which regenerated tubular dentin tissue [40]. Following neural inductive culture, SHEDs presented higher expression levels of neuronal and glial cell markers than hDPSCs, such as $\beta$-III-tubulin, tyrosine-hydroxylase (TH), microtubule-associated protein 2 (MAP2), and Nestin. Dopaminergic (DAergic) neurons could be produced by SHED-derived neurospheres in a DAergic induction system [41]. Additionally, SHEDs could act as neuroprotector agents to promote neural functional recovery through paracrine effects and inhibit glial scar formation after spinal cord contusion [42]. Conditioned media (CM) derived from SHEDs, containing various neurotrophic factors, enhanced peripheral sciatic nerve regeneration with axon regeneration and remyelination, which improved motor functions thus preventing muscle atrophy [43]. These multifaceted neural regeneration activities render SHEDs as an optimal cellular source to improve the injured nerve. For osteogenic potential, SHEDs induced new bone formation in vivo by recruiting host osteogenic cells, rather than differentiating into osteoblasts which happened in vitro [20]. Larger osteoids and more collagen fibers were formed by SHEDs with polylactic-coglycolic acid (PLGA) membrane transplantation as compared to DPSCs and BMMSCs [44].

In addition to striking multi-lineage differentiation potential, the immunomodulatory effects have been reported in MSCs from dental pulp tissue, which may function through correcting the underlying pathological immune responses. hDPSCs have been suggested to regulate local immune response by suppressing the expression of metalloproteinases (MMPs) including MMP3 and MMP13 and to inhibit acute rejection of allograft by releasing transforming growth factor-beta (TGF- $\beta$ ) [45]. Dai et al. found that SHEDs suppressed the CD4+ T cell-driven responses via inhibiting the proliferation of $\mathrm{T}$ lymphocytes and the upregulated ratio of Th1/Th2 by inducing the expansion of Treg cells [46]. Local injection of SHEDs increased the number of anti-inflammatory CD206+ M2 macrophages and altered the cytokine expression profiles in periodontal tissues with periodontitis [47].

2.2. Stem Cells from the Apical Papilla (SCAPs). During tooth development, dental papilla derived from the ectomesenchyme ultimately converts into the dental pulp tissue and migrate to locations around the apex [48]. Root development and apical closure could still be observed in immature permanent teeth suffering from periapical periodontitis or abscess. These clinical phenomena suggested that a population of MSCs might reside in apical papilla. SCAPs were isolated from third molar root apical papilla, which contains fewer blood vessels and cells than dental pulp tissue [21, 48].

SCAPs have shown a greater potential to regenerate dentin than DPSCs, since they have higher proliferation with greater telomerase activity, suggesting that SHED is a cell source for odontoblasts responsible for the production of dentin [21]. Previous studies have confirmed that SCAPs are capable of differentiating into odontoblast-like cells and form a typical dentin-like structure on the surface of HA/TCP [21, 48]. Larger areas of mineralized nodules positive to Alizarin Red were formed by SCAPs with culture medium containing L-ascorbate-2-phosphate [48]. When SCAPs mixed with host cells, odontoblasts positive for dentin sialophosphoprotein (DSPP) and dentin matrix protein 1 (DMP1) and ectopic formation of vascularized pulp-like tissue were detected in mice molars [49]. A greater migration ability assessed by scratch assay enhanced their capacity for dentin regeneration by cell homing [21]. Considering their role in root development, SCAPs have been suggested to possess a significant potential for root regeneration. A functional bioroot with periodontal ligament tissue was generated in the alveolar socket of a minipig following transplantation of human SCAPs and periodontal ligament stem cells (PDLSCs). Additionally, SHEDs showed a PDL-related marker in vitro and exhibited greater mineralization capacity on account of higher expression levels of ALP, bone sialoprotein (BSP), and OCN expression compared to PDLSCs [50]. Therefore, SCAPs have been considered as a promising alternative source for periodontal tissue regeneration. Their potential for angiogenesis has also been confirmed in 3Dprinted HA scaffolds. Derived from the cranial neural crest, SCAPs possess neurogenic differentiation potential similar to DPSCs and SHEDs. After transplantation of the human apical papilla tissue into the injured spinal cord in rats, improvements were observed in gait and glial reactivity [51]. Besides, SCAPs may be a potential immunotherapeutic tool for immunological diseases due to their low immunogenicity and capability of inhibiting $\mathrm{T}$ cell proliferation [52].

2.3. Periodontal Ligament Stem Cells (PDLSCs). Periodontal ligament (PDL) is a soft connective tissue, which contains progenitor cells that maintain tissue homeostasis and 
regeneration of periodontal tissues [22, 53]. PDLSCs were isolated from the attached PDL of the extracted third molar with expression of two early MSC markers STRO-1 and CD146/MUC18 and higher levels of scleraxis, a tendonspecific transcription factor, compared to DPSCs [22].

The potential for the cementogenic/osteogenic differentiation of PDLSCs has been shown by the formation of calcified nodules and expression of ALP, matrix extracellular protein (MEPE), BSP, OCN, and TGF- $\beta$ receptor I [22]. Human PDLSCs have been demonstrated to be a reliable source for the fabrication of 3D PDL tissues [54]. Typical cementum/PDL-like structures, including Sharpey's fiberlike tissue, were generated after the transplantation of human PDLSCs into the rat periodontal lesion sites [22]. PDLSCs also contribute to root regeneration. When combined with SCAPs, they generated a collagen fiber which anchored into the newly formed cementum on the surface of the HA/TCP carrier, and formed a functional root supporting a porcelain crown [21]. Extracellular vesicles (EVs) released by PDLSCs have also been reported to possess osteogenic properties and promote bone regeneration. Collagen membranes with PDLSC-EV transplantation showed osteoid formation with an osteoblast-like structure on the host native bone side and new bone irregularly arranged in the implant site of rats subjected to calvarial defects. Neural crest-derived PDLSCs spontaneously express neural protein markers as Nestin and growth associated protein-43 (GAP-43) upon xeno-free culture conditions [55]. In addition to previously demonstrated adipocytes and chondrogenic cells, several studies sequentially reported the differentiation potential of PDLSCs toward endothelial cells, cardiac myocytes, islet-like cells, and retinal ganglion-like cells [11]

Based on immunosuppression, low immunogenicity, and the ability to produce a vast array of cytokines, PDLSCs and their products have the potential to treat inflammatory disorders and autoimmune diseases. Ding et al. found the PDLSCs failed to express HLA-II DR and costimulatory molecules and possessed marked immunosuppression via PGE2induced T-cell anergy [56].

2.4. Gingival-Derived Mesenchymal Stem Cells (GMSCs). A population of progenitor cells or stem cells has been identified in the spinous layer of human gingiva, an easily accessible tissue from remnants or discarded tissues following routine dental procedures, namely GMSCs.

The osteogenic differentiation of GMSCs was determined by formed deposits with positive Alizarin Red S staining and upregulated expression of OCN in vitro, while their ability for osteogenic differentiation has not been observed in vivo [23]. However, recent evidence has shown that EVs derived from GMSCs exhibit significant osteogenic properties as revealing high expression levels of RUNX2 and BMP2/4 and abundant extracellular matrix (ECM) and nodules of new bone formation [57]. Transplantation of GMSCs formed connective-like tissues expressing collagen I, which is absent in DPSCs or PDLSCs [23]. Human fetal GMSCs have an ability for gingival differentiation automatically in vivo because they may contain more precursor cells to differentiate into gingival cells. After having been transplanted into the gingiva defects of rats, human GMSCs generated new tissue like normal gingiva [58]. GMSCs are capable of neurogenic differentiation since they are positive for glial fibrillary acidic protein (GFAP), neurofilament (NF-M), and $\beta$-tubulin III upon neural differentiation conditions [23]. GMSC spheroids have shown differentiation potential into both neuronal and Schwann-like cells with a 3D-collagen scaffold. And 3D bioprinted grafts with GMSCs formed nerve tissue with a normal size at the defect of rat facial nerves and showed higher therapeutic potential on facial palsy. These findings have demonstrated that GMSCs present promising potential for nerve regeneration and functional recovery [59]. Besides, they could also differentiate into adipocytes, chondrocytes and endothelial cells $[23,60]$.

Importantly, GMSCs have distinctive immunomodulatory functions, as they could suppress peripheral blood mononuclear cells (PBMCs) and upregulate IFN- $\gamma$-induced indoleamine 2,3-dioxygenase(IDO) and IL-10. Spheroid-derived GMSCs displayed the capability to enhance the secretion of several chemokines and cytokines and improved resistance to oxidative stress-induced apoptosis. They have been reported to attenuate chemotherapy-induced oral mucositis [61].

2.5. Dental Follicle Stem Cells (DFSCs). The dental follicle (DF) is responsible for forming alveolar bone and the root-bone interface in tooth development; it is an ectomesenchymal tissue that contains progenitor cells (PCs) for periodontal ligament cells, cementoblasts, and osteoblasts $[24,25,28]$. These PCs were isolated from the dental follicle of the extracted third molars, characterized by expressed undifferentiated cell markers Notch-1 and Nestin, namely DFSCs $[24,25]$.

DFSCs express a higher level of insulin-like growth factors (IGF-2) compared to hMSCs and exhibit higher proliferation potential and colony-forming ability compared with SHEDs, DPSCs, and PDLSCs, suggesting their potential in regenerative medicine $[24,25,62,63]$. Their superior osteogenic properties have been reported by several studies. DFSCs show higher expression levels of osteogenic-related markers such as RUNX2 and ALP compared to SHEDs and DPSCs [62]. Long-term culture of DFSCs with differentiation inductive medium have demonstrated that they have the potential to differentiate into osteoblasts expressing BSP and OCN and form calcium deposits [24, 25]. DFSCs are more immature than PDLSCs. There is less heterochromatin in the nucleus and fewer organelles and bundles of microfilaments in the cytoplasm of DFSCs than in the cytoplasm of PDLSCs on ultrastructural comparison [63]. The higher expression of DSPP in DFSCs has shown its preferable potential for odontogenic differentiation and dentin regeneration compared to PDLSCs. And they generated complete dentin including dentin, predentin, and calcospherites upon the induction of treated dentin matrix (TDM) [11]. The properties of periodontal differentiation of DFSCs have also been demonstrated. They are able to form fibrous membrane PDL-like structures or calcified nodules with bone- or cementum-like structures under in vitro conditions, suggesting their potential for periodontal differentiation [24, 25]. Upon in vivo transplantation, DFSCs derived from the apical 
end of human developing root could produce a cementum/PDL-like complex characterized by a thin layer of cementum-like tissues and PDL-like collagen fibers inserted perpendicularly into the newly formed cementum-like deposits [25]. These findings suggest that DFSCs are a promising alternative source for bioroot engineering.

Furthermore, the immunomodulatory effects of DFSCs also favor their therapeutic potential to treat autoimmune, inflammatory, and allergic diseases. Compared with SHEDs and DPSCs, DFSCs stimulated by IFN- $\gamma$ remarkably increased the number of CD4+FOXP3+ Treg cells and suppressed the proliferation and apoptosis of peripheral blood mononuclear cells (PBMCs) $[62,64]$.

2.6. Tooth Germ Stem Cells (TGSCs). In the bell stage, tooth germ consists of three components including enamel organ, dental mesenchymal cells (dental papilla or pulp), and dental follicle. The progenitor cell populations of dental mesenchymal cells, named TGSCs, have been isolated and identified from human third molar tooth germ [26]. The expression of DSPP has confirmed the odontogenic differentiation of TGSCs with the treatment of BMP2 and BMP7 [65].

The osteogenic differentiation capability of TGSCs has been demonstrated, as new bone formation was obtained in the pore area of the HA/TGSC implants. TGSCs have the potential to regenerate cartilage tissue, which is attributed to their chondrogenic differentiation ability upon induction. After TGSCs attached to 3D biological scaffolds, abundant hyaline cartilage-specific extracellular matrix (ECM) and collagen type II expression were found [66]. TGSCs were also able to differentiate into hepatocytes under hepatic induction. This was indicated by the expression of the liver-specific albumin gene, positive staining for albumin protein, and morphological change [26]. In rats with injured liver, transplantation of differentiated TGSCs could suppress the liver hydroxyproline content and reduce areas of damage, therefore suppressing liver fibrosis and steatonecrosis [26], suggesting that TGSCs are useful in cytotherapy for liver diseases.

\subsection{Alveolar Bone-Derived Mesenchymal Stem Cells} (ABMSCs). BMMSCs have been isolated from various bone tissues such as the ilium by an invasive procedure, namely, marrow aspiration. Alternatively, collecting ABMSCs from alveolar bone during the course of dental surgery is providing a new isolation method with a few extra invasive interventions [27]. ABMSCs have favorable osteogenic differentiation potential comparable to BMMSCs but a weaker potential to differentiate into chondrocytes or adipocytes [27]. New bone has been detected in a rabbit critical-size mandibular bone defect model with transplants which consist of ABMSCs and $\beta$-TCP [67]. ABMSCs also have the potential for bone tissue regeneration, and their potential to reconstruct alveolar bone will contribute to improving periodontal defects.

\section{Dental MSC-Based Therapy for Dental Diseases}

Considering the multi-lineage potential, dental MSCs are suggested as promising cells for the treatment of dental dis- eases. Therefore, there have been a variety of therapeutic applications in dental medicine, ranging from preclinical studies (Table 1) to initial clinical trials (Table 2).

3.1. Endodontic Diseases. Dental caries and tooth trauma are common diseases associated with the teeth, which destroys the rigid structure of the teeth, both the enamel and dentin, resulting in pulp necrosis and periapical disease. For mature permanent teeth, the current routine clinical treatment is traditional root canal therapy based on pulpectomy, which involves the removal of damaged dental pulp tissue, enlargement of the root canal, and filling of the sterile canal with artificial filling materials [68]. When immature permanent teeth suffer from necrotic pulp/apical periodontitis, tooth development would be arrested, resulting in immature teeth with a thin root dentin and open apices. These teeth need to be treated with special measures based on pulpectomy, including the traditional apexification procedure and an apical mineral trioxide aggregate (MTA) plug [32]. Despite the wide implementation of the current routine treatment, the lack of biological dentin/pulp or dentin-pulp complex and the limitations of existing materials may lead to a great risk of serious reinfection and tooth fracture, thereby resulting in a poor survival rate for teeth. Therefore, the biological regeneration of dentin and pulp could be an ideal and alternative solution to replace defective dental structures in modern dental medicine. Based on different pulp conditions, several novel ideas for dentin-pulp complex and dental pulp regeneration therapy are presented [32]. Firstly, for local regeneration of the dentin-pulp complex following pulpotomy, combining dental MSCs with growth factors or platelet-rich plasma (PRP) is a promising solution to induce DPSCs and capillaries from the residual root pulp tissue and regenerate dentin tissue. Secondly, for a complete regeneration of the dentin-pulp complex for devital tooth after pulpectomy or pulp necrosis, cell homing and cell transplantation are utilized to achieve regeneration of the entire dental pulp for adult permanent teeth or revascularization for immature permanent teeth. However, the traditional revascularization approach fails to regenerate the dentinpulp complex, unlike novel tissue engineering [68]. With various types of stem cells identified and remarkable breakthroughs in tissue engineering, numerous researches on dental MSC-mediated dentin and dental pulp regeneration have been carried out in animal models and human clinical trials [32].

3.1.1. Dentin Regeneration. The composite construct made up of porcine SHEDs and $\beta$-TCP scaffold has been directly capped on the created chamber roof defects in the premolars of swine, showing that almost complete dentin regeneration was observed with the newly formed dentin-like structure performing sparse porosity and certain thickness. It is indicated that the novel therapy based on dental MSCs significantly regenerated the dentin-like structure and is useful in direct pulp capping [69]. Subsequent research explored hDPSCmediated dentin regeneration. hDPSCs were cultured onto the human dentin treated by ethylene diamine tetra-acetic acid and citric acid (hTD) and then implanted in the mouse model. 


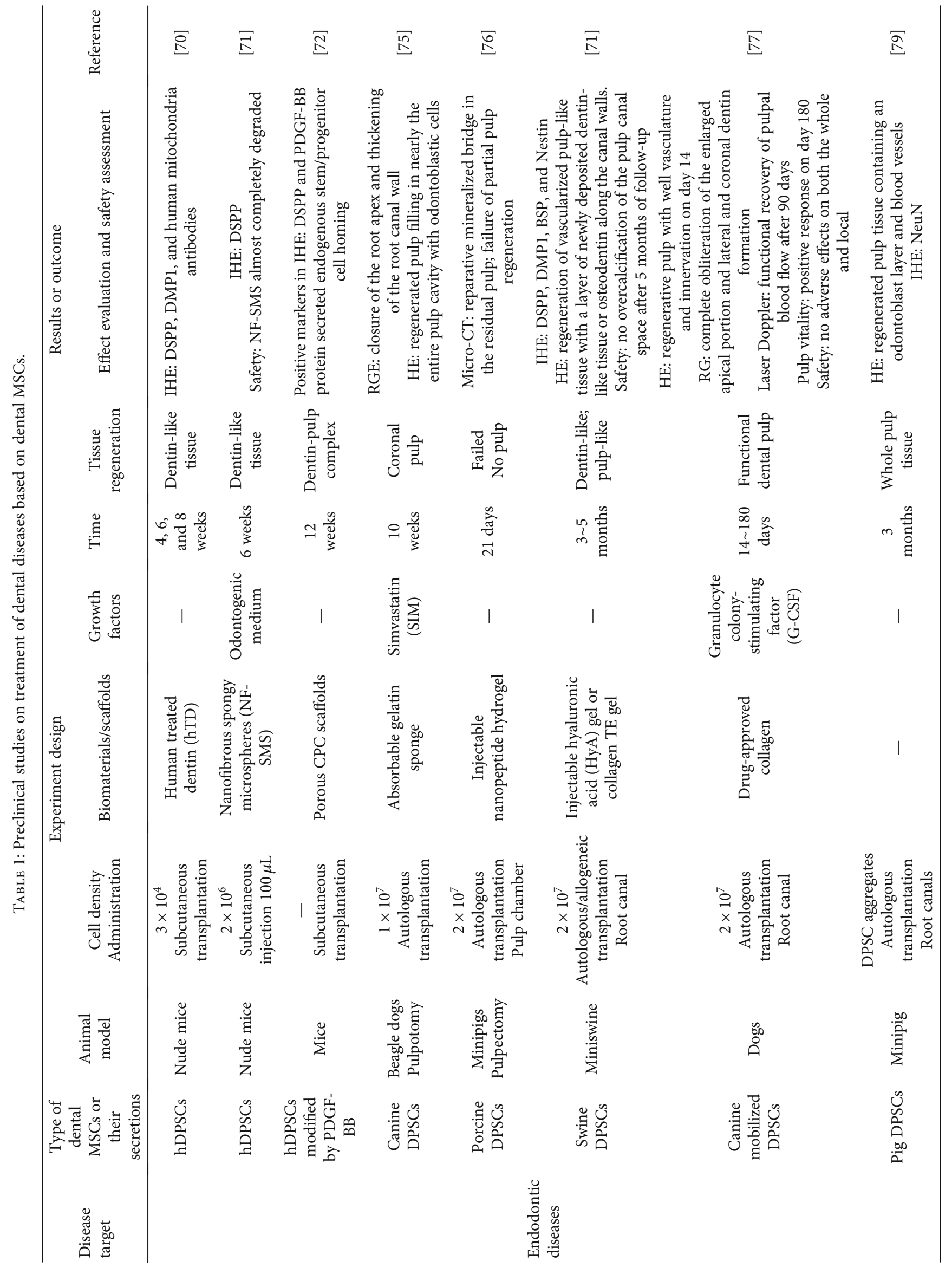




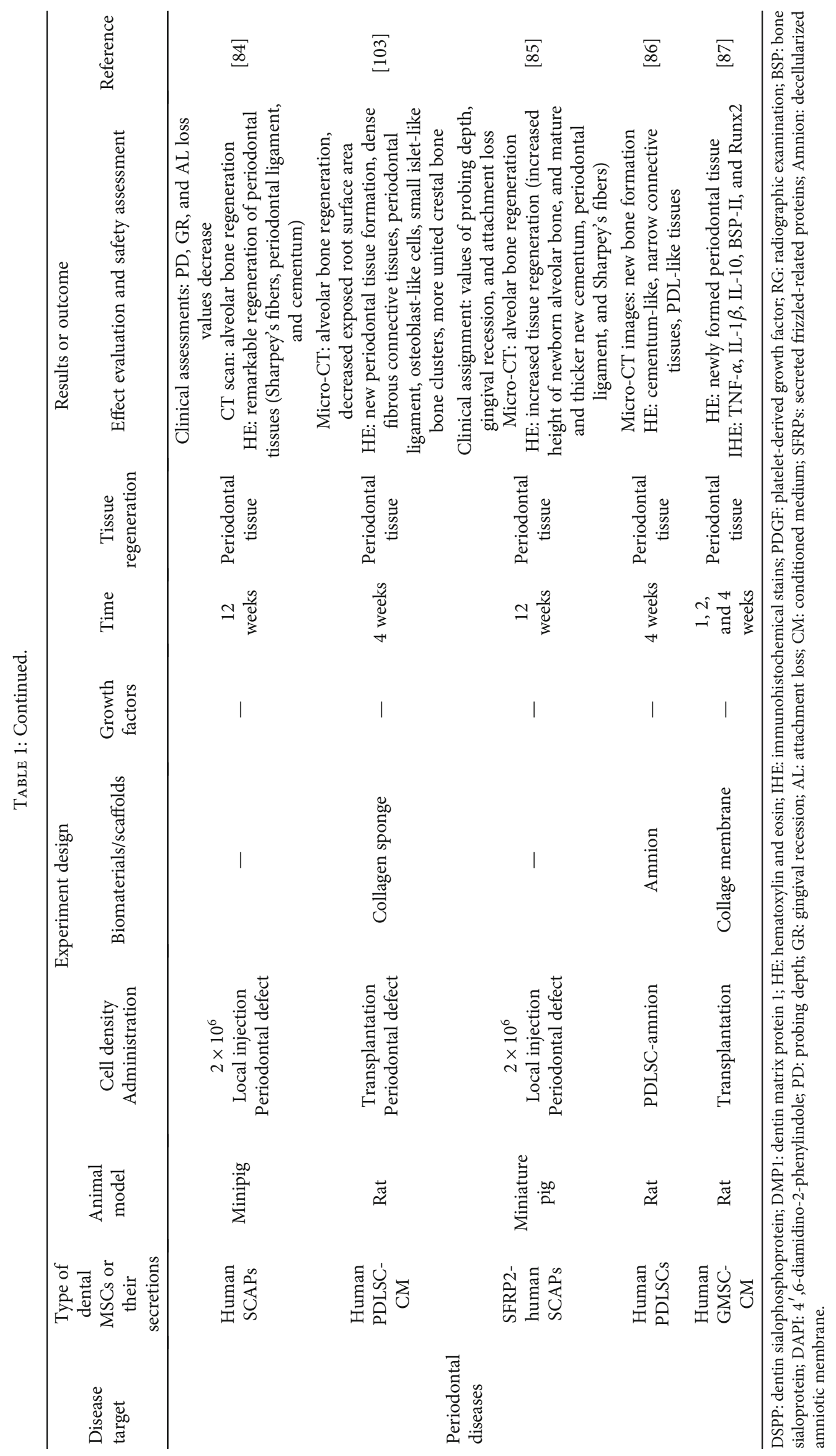




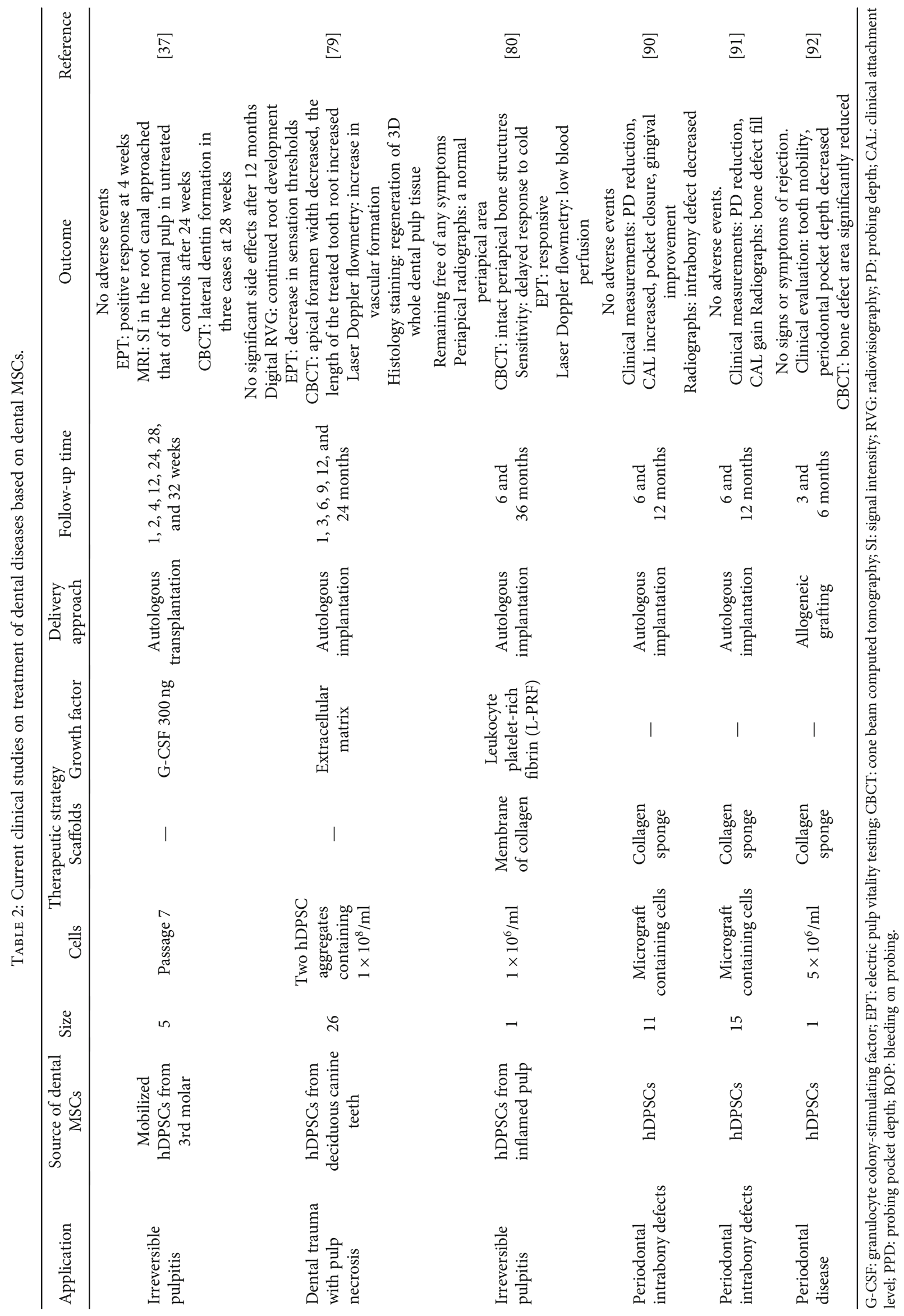


Formation of dentin-like tissues expressing specific dentin markers demonstrated that hDPSCs could be induced by hTD to regenerate the complete dentin tissue in vivo [70]. Meanwhile, hDPSCs were seeded onto a novel injectable cell carrier named nanofibrous spongy microspheres (NF-SMS). The result showed that a supported dentin-like tissue was generated in nude mice [71]. However, the narrow root foramen/limited tissue infiltration and blood supply would hold back the application of dental MSCs in clinic. A combination of some powerful growth factors and stem cells could serve as an alternative solution. Zhang et al. modified hDPSCs by overexpressing platelet-derived growth factor- (PDGF-) BB, which is a potent mitogenic factor as a mediator in wound healing and tissue repair, and obtained more dentin-like mineralized tissue similar to tooth dentin tissue in vivo. Further studies demonstrated that PDGF-BB-modified hDPSCs facilitated stem cell homing via the PI3K/Akt pathway and improved hDPSC-mediated dentin-pulp complex regeneration [72]. Lastly, a novel dentin-pulp-like organoid was developed by constructs mixed with hDPSCs and Matrigel in an odontogenic differentiation medium. The organoid demonstrated a biologically active response to biodentine supplements and suggested hDPSCs as a future approach for tooth regeneration [33]. Although scientific evidence shows a positive trend to dentin regeneration using dental MSCs, especially hDPSCs in animal models, there is a lack of persuasive evidence of clinical trials up to now.

3.1.2. Pulp/Dentin-Pulp-Like Regeneration. Dental pulp plays an indispensable role in maintaining homeostasis of the tooth, but its capacity of self-repair is highly limited. Hopefully, recent preclinical and clinical studies on cell homing and autogenous/allogeneic dental MSCs transplantation have provided further evidence of dental pulp regeneration. Depending on the clinical situation, there are mainly two cell-based pulp-regeneration strategies, partial dental pulp regeneration and whole pulp tissue regeneration [73]. A tentative experiment achieved rat DPSC-mediated partial dental pulp regeneration in rat molars after pulpotomy, suggesting that the remaining healthy pulp tissue could be recoverable and may have the potential to regenerate the lost portion of the dental pulp tissue [74]. Subsequent researches were performed in large animal models to test the feasibility of this regenerative approach. In a beagle dog model, Jia et al. transplanted canine DPSCs (cDPSCs) pretreated with simvastatin into immature premolars treated by pulpotomy. Then, regenerated coronal pulp was found filling nearly the entire pulp chamber with newly formed dentin and odontoblastic cells seen in the regenerated area, suggesting that coronal dental pulp regeneration could be realizable by cDPSCs transplantation [75]. Nevertheless, a study implanted pDPSCs/hydrogel into premolars and molars after pulpotomy in a minipig model and only found reparative dentinogenesis without dental pulp regeneration, highlighting the necessity for further investigations to develop a favorable regenerative microenvironment [76].

In recent years, there is growing concern about cell-based regenerative therapy for pulpless teeth. Moreover, several clinical studies are currently underway to confirm the efficacy and safety of stem cell-based regenerative therapy. Inspiring outcomes have been reported for the whole dental pulp regeneration. Nakashima et al. developed a composite of drug-approved collagen scaffold and clinical-grade human mobilized DPSCs (MDPSCs) induced by granulocyte colony-stimulating factor (G-CSF) and achieved complete dental pulp regeneration by autologous transplantation with the composite in the mature teeth of dogs after pulpectomy. Similar to the healthy dental pulp tissue, regenerative pulplike tissue presented good vasculature, innervation, odontoblast-like cells, and recovered function. Moreover, rare adverse effects confirmed the safety of cell therapy for dental pulp regeneration. A notable finding is that there were no significant age-related changes in biological properties and the stability of human MDPSCs in vitro and in vivo [77]. Then, this team performed a pilot clinical study to further demonstrate the availability and clinical safety of autologous transplantation of MDPSCs in pulpectomized teeth [37]. Functional dentin formation was observed by cone beam computed tomography (CBCT) in three of the five patients. Further study showed that varisble sizes hDPSCs constructs possess the ability of self-organizing and can fill the human tooth root canal to regenerate blood vessel-rich pulp-like tissues after implantation in the subcutaneous space of mice [78]. Much more significantly, whole functional dental pulp tissue regeneration in a minipig was observed after pig DPSC aggregates were implanted into young permanent incisors. And newly formed dental pulp tissue containing an odontoblast layer and blood vessels as well as the expression of neuron markers NeuN indicated that functional dental pulp regeneration could be achieved in a large preclinical animal model [79]. Recently, Xuan et al. performed a randomized clinical controlled trial for treating immature permanent teeth injuries due to trauma. Taking apexification as a control group, this study demonstrated that not only could hDPSCs implantation regenerate 3D dental pulp tissue with blood vessels and sensory nerves but it could also show better efficacy and safety of hDPSCs implantation [79]. The majority of MSC-based endodontic treatments were performed in immature permanent teeth of adult patients. Interestingly, a recent case showed a personalized cell therapy in tooth $\# 28$ with symptomatic irreversible pulpitis in a 50-year-old man. As reported, hDPSCs were isolated from the inflamed dental pulp tissue of the diseased tooth \#28. Combined with leukocyte platelet-rich fibrin (LPRF) from the patient's blood, expanded hDPSCs were introduced into the prepared root canal. There was a positive response to an electric pulp test and a vitality test after a follow-up period of 36 months, which indicated that this MSC-based therapeutic method contributed to denetal pulp regeneration [80].

3.2. Periodontal Diseases. Periodontitis leads to the damage of periodontal tissue including gingiva, cementum, ligament, and alveolar bone [81]. At present, periodontitis is routinely treated by debridement, surgery involving mechanical means, and guided tissue regeneration (GTR), which remain unsatisfactory due to rare regeneration [82]. The ultimate therapeutic goal for periodontal diseases is to regenerate lost 
periodontal tissues. To address this, cell-based tissue regeneration has become one of the optimal periodontal therapies [81]. Several outstanding reviews have summarized the progress of cell-based regeneration of periodontal tissues [82, 83]. In the current review, we focus on the advance of dental MSC-based therapy for periodontal diseases, particularly periodontitis.

Previous studies of dental MSC-based therapy for periodontal tissue regeneration mainly focus on PDLSCs and DPSCs, but the source of PDLSCs is limited. A recent study discovered that SCAPs could serve as an alternative cell source for periodontitis treatment. Human SCAPs were injected subperiosteally to the surface of bone around the periodontitis defects in minipigs. It demonstrated that local injection of SCAPs improved gingival status and enhanced both bone and cementum regeneration [84]. With the discovery of key factors that maintain the function of SCAPs in periodontal treatment, subsequently, a strategy of gene modification has been studied by Li et al. By comparative investigation, they found that SCAPs overexpressing with SFRP2 promoted SCAP-mediated bone, PDL, and cementum regeneration in a minipig periodontitis model [85]. Recently a novel method named cell transfer technology was devised, in which cells were transferred onto a scaffold surface. With this new approach, Iwasaki et al. transferred human PDLSCs to the decellularized amniotic membrane (amnion) and transplanted the PDLSC-amnion into a rat with a created dehiscent-type periodontal defect. Newly generated cementum, PDL, and bone were detected, suggesting dental MSC-based treatment as a proposed new technology for periodontal diseases [86]. Conditioned medium generated by dental MSC culture (dental MSC$\mathrm{CM}$ ), which contains growth factors, cytokines, and other active substances, is considered as another new trend in periodontal tissue regeneration. Cell-free dental MSC-CM is more convenient and safer to apply in clinic than cellbased therapy. Qiu et al. transplanted collagen membranes loaded with concentrated GMSC-CM and PDLSC-CM into the buccal periodontal defects of molars in rats. More newly formed periodontal tissues were observed in both GMSC-CM and PDLSC-CM [87].

Following supporting evidence provided by numerous animal studies, the first human clinical trial was carried out to treat periodontal osseous defects in three patients through autologous ex vivo PDLSCs transplantation [88]. Later researchers devised a novel approach with stem cell assistance in the periodontal tissue regeneration technique (SAIPRT) bypassing ex vivo PDLSCs. In a case report, researchers transplanted the transferable mass consisting of gelatin sponge and soft tissue harboring PDLSCs scraped from cementum and the alveolar socket of the third molar into the intrabony defect of another molar in the same patient. Then, they obtained clinical success with the reduction of probing pocket depth and the recovery of attachment over the evaluation of one year. Although the study is not certain about the number and viability of immediate PDLSCs transplantation, SAI-PRT might be a constructive avenue in the treatment of periodontal osseous defects [89]. Meanwhile, Aimetti et al. reported serial cases to explore the clinical potential effects of the application of hDPSCs to treat deep intrabony defects via regenerative therapy. A total of 11 periodontitis patients with intrabony defects received treatment including a minimally invasive flap and autologous hDPSCs loaded on a collagen sponge. Significant clinical improvements and rare adverse effects were observed in a one-year follow-up [90]. Then, the team performed a randomized controlled clinical trial to evaluate the effectiveness of the novel therapeutic strategy as studied above. A remarkable reduction of probing depth (PD), a gain of clinical attachment, and the filling of bone defects in a test group further suggested that this cytotherapeutic approach based on PDLSCs engineering is a safe and innovative strategy to treat severe periodontal defects [91]. Moreover, a case report presented the effect of allogeneic hDPSCs transplantation in periodontal tissue regeneration of an aged periodontitis patient. hDPSCs were obtained from the dental pulp tissue of a 7-year-old donor and expanded. During periodontal surgery of the mesial circumferential bone defect, hDPSCs were seeded into a lyophilized collagen-polyvinylpyrrolidone sponge. After the allogeneic graft, the patient exhibited improved clinical manifestation without any sign of rejection. It is indicated that allogeneic hDPSCs transplantation could induce periodontal tissue regeneration [92].

\subsection{Therapeutic Strategies in MSC-Based Dental Medicine.} Due to their excellent potential for multil-ineage differentiation, dental MSCs are considered as an ideal source for tissue engineering and regenerative dental medicine. To date, researchers are looking for a feasible, safe, and effective approach for regenerative and translational dentistry [16]. Three feasible regenerative strategies based on dental MSCs have been proposed to treat dental diseases in clinic (Figure 2).

3.3.1. Scaffold-Supported Tissue Engineering. Generally, the principles of tissue engineering are based on three elements, including stem cells with multi-lineage differentiation potential, scaffolds as carriers for stem cells, and bioactive molecules inducing differentiation [93]. Dental MSCs are regarded as ideal cells for dental tissue engineering since they possess a shared embryological origin with craniofacial tissue [94]. Biocompatible scaffolds provide a favorable 3D microenvironment for stem cells, which regulate proliferation and differentiation [16]. In regenerative dentistry, current dominating attempts and studies of scaffold-supported tissue engineering include regeneration of dentin, dental pulp, and periodontal tissue and formation of bioroot. In a miniswine model, Zhu et al. transplanted autologous and allogeneic swine DPSCs carried by a bioscaffold hydrogel into the root canal space of the miniswine. Orthotopic vascularized pulplike tissue regeneration was achieved with newly generated dentin-like tissue or osteodentin along the canal walls [95]. Similarly, a study used a root-shaped HA/TCP scaffold with allogeneic swine DPSCs, which was wrapped by a vitamin C-induced allogeneic PDLSC sheet and implanted into the jaw bone socket in swine and successfully regenerated a functional bioroot with a dentinal tubule-like structure and a functional PDL-like structure after six months [96]. Recently, a cell-laden hydrogel encapsulating GMSCs was used to 

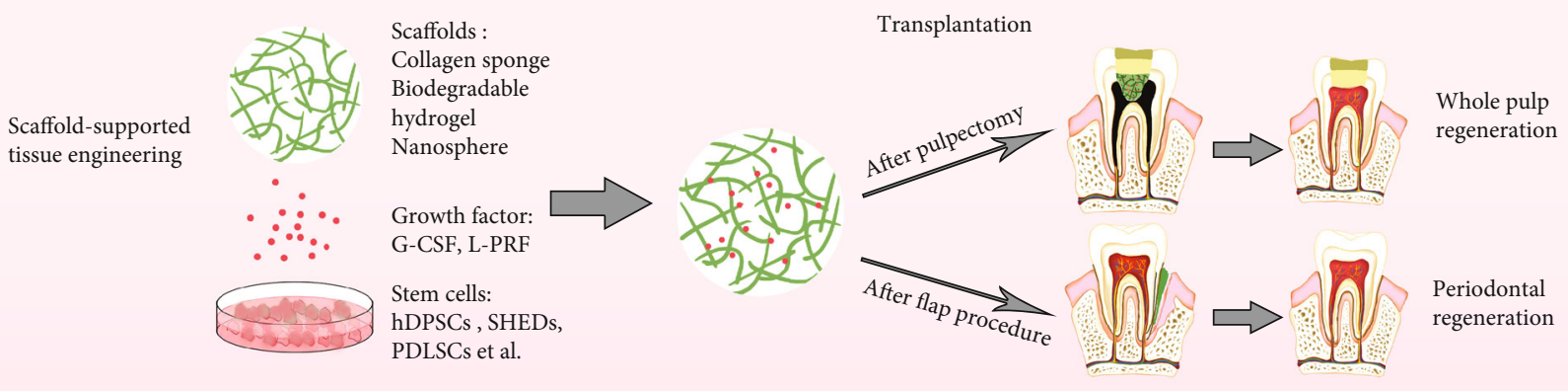

(a)
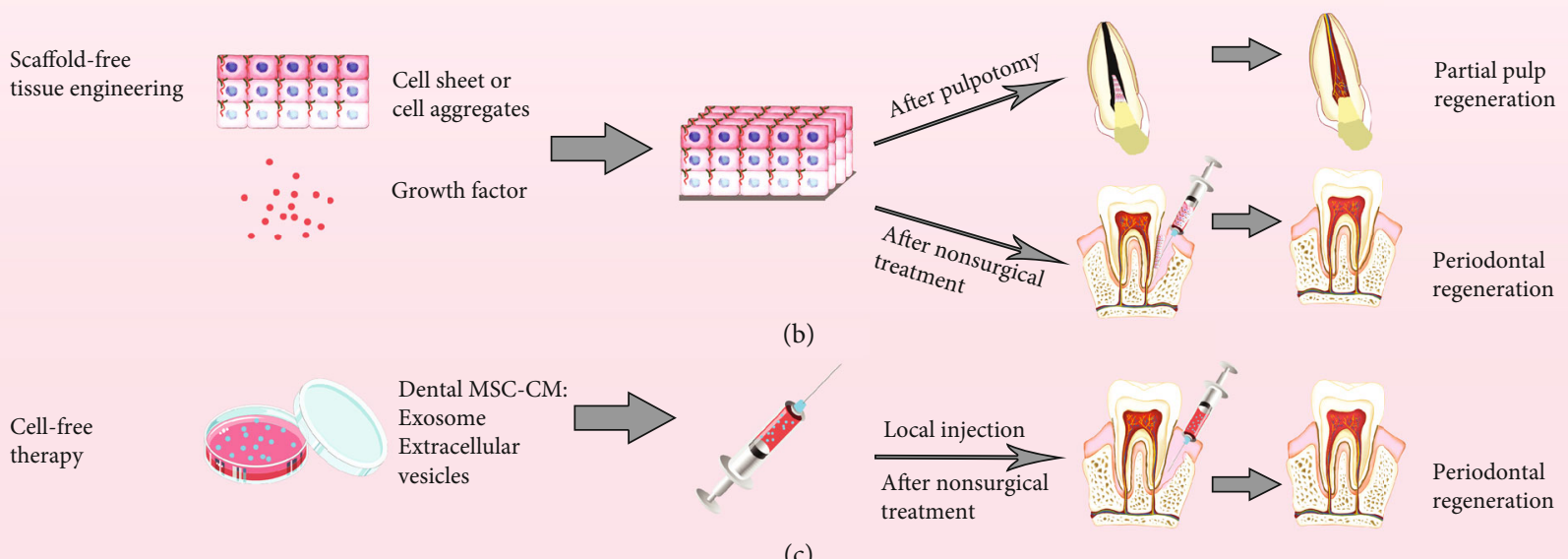

Periodontal regeneration

FIgURE 2: Three therapeutic strategies of endodontic and periodontal diseases using dental MSCs. (a) Dental tissue regeneration through a classic tissue engineering model, consisting of dental MSCs, supporting biomaterial scaffolds, and growth factors. (b) Dental tissue regeneration by tissue engineering without scaffolds. (c) Dental tissue regeneration with a cell-free approach using conditioned medium (CM) with exosomes and/or extracellular vesicles (EVs) secreted by dental MSCs.

promote craniofacial bone tissue regeneration. This study showed complete bone regeneration around ailing dental implants in rat peri-implantitis [97]. To achieve dentinepulp complex regeneration, the optimal protocols should integrate cells, biomaterials, and growth factors. However, there are still several issues related to long-term safety and effectiveness, such as host immune rejection, degradation, and potential infection.

3.3.2. Scaffold-Free Strategies for Tissue Engineering. Based on the formation of tridimensional cell-to-cell aggregates without any other external support, scaffold-free technologies avoid the unknown risks of using biomaterials. Two scaffold-free strategies have caught the eye of researchers, which are cell sheets and cell injection. As a unique method of cell processing via culturing in temperature-responsive cell culture dishes or in ascorbic acid, cell sheets have been widely explored and applied in regenerative dentistry [16, 98]. PDLSC cell sheets were autologously transplanted into the denuded root surface in a canine model with a one-wall intrabony defect, and periodontal tissue regeneration was remarkably observed with both cementum and PDL fibers after eight weeks [99]. Cell injection might be a common treatment for periodontal disorders because it is a minimally invasive process. Local injection of allogeneic SCAPs has been shown effective for treating periodontitis by the promotion of periodontal tissue regeneration in a miniature pig model [84]. However, the cell injection approach, independent from the use of any scaffold or biomolecule, has some practical issues, for instance, the risk of losing cell properties in the asepsis storage period and a small application range.

3.3.3. Cell Homing or Cell-Free Therapy. Cell homing is a cellfree approach to repair or regenerate tissue through active recruitment of host endogenous cells to the injured region, mainly via bioactive molecules. Compared with stem cell engraftment, cell homing may evade many hurdles in clinical translation of cell transplantation, including tumorgenicity, antigenicity, host rejection, and infection associated with cell-based therapies [100]. Exosome secreted by dental MSCs could act as paracrine signalers in cell homing. The exosome is one of EVs containing cytokines and microRNAs and plays a vital role in stem cell-based therapy by releasing molecules in target tissues [16]. Furthermore, dental MSCs may provide the secretome/CM with future regenerative therapeutic applications. Compared with the therapy using dental MSCs, dental MSC-CM, which is cell free, exhibits remarkable biological properties, including higher safety, migration activity, and greater ability of odontoblastic differentiation [101]. In a recent work, PDLSC-CM was transplanted into surgically created periodontal defects in a rat, and it was found that PDLSC-CM containing extracellular matrix proteins, enzymes, angiogenic factors, growth factors, and 
cytokines enhanced periodontal regeneration by suppressing the inflammatory response via TNF- $\alpha$ production [102]. The dental MSC-mediated cell-free therapeutic approach is an appealing approach for treating dental diseases and has predominance over cell-based therapy despite some limitations in it. The bioactive molecules involve secretomes released by various populations, and their mechanisms need to be further understood.

\section{Dental MSC-Based Therapy for Nondental Diseases}

4.1. Other Oral Diseases. The present therapeutic application of dental MSCs is not limited to endodontic and periodontal diseases. Recently, dental MSC-based therapy for other oral diseases has been proposed in animals and humans, such as craniofacial bone defects, progressive temporomandibular joint (TMJ) arthritis, facial nerve lesions, taste bud loss, and Sjogren's syndrome.

The craniofacial bone defect could be repaired by bone regeneration with dental MSCs [96, 103]. In a wellestablished rat model with peri-implantitis, GMSC-laden adhesive alginate hydrogels were injected into the bony defect sites around implants, which increased implant survival and the amount of recovered bone [96]. Dental MSCs also hold promise for the treatment of facial nerve injury. Recent experimental evidence showed that a novel method to treat crush injury of rats' facial nerve is via a single application of human SHEDs immediately, which could promote a positive local effect on neuroprotection and remyelination in 2 weeks [104].

A further important application of dental MSCs is for the treatment of TMJ disorders. Common TMJ arthritis often leads to sustained synovitis, cartilage and bone destruction, and pain. Considering the potential immunomodulatory features of human DPSCs, Cui et al. tried to locally inject hDPSCs into the articular cavity to treat rat TMJ arthritis. It was found that DPSCs relieved hyperalgesia and synovial inflammation, attenuated cartilage and matrix degradation, and promoted bone regeneration [105]. Dental MSCs have been suggested to promote taste bud regeneration and have promising potential applications in postsurgery tongue reconstruction of patients with tongue cancer [106]. Interestingly, recent work has also suggested that SHEDs exert a protective effect on the secretory function of the salivary gland and exhibit therapeutic potential for the improvement of hyposalivation in Sjogren's syndrome [107].

4.2. Extraoral Diseases. Besides widespread application for treating oral diseases, as a powerful autologous stem cell source, dental MSCs also have great therapeutic potential for the treatment of multiple systemic ailments. A recent review has summarized the extensive usage of hDPSCs in the cell-therapeutic paradigm shift to treat various diseases [18]. Other dental MSCs have also been applied in the treatment of extraoral diseases like neurodegenerative diseases and autoimmune and orthopedic disorders. Dental MSCs have a remarkable potential to treat neural diseases such as spinal cord injury (SCI) and peripheral nerve injury, like sci- atic nerve and superior laryngeal nerve (SLN) injury, owing to their ability to differentiate into neural-like cells and regenerate neural tissue [107]. SCI is a severe traumatic central nervous system disease resulting in the damage of sensory and motor functions. It has been demonstrated by recent studies that dental MSCs could facilitate functional improvement after SCI in animal models [108-111]. For instance, SHED-CM loaded in collagen hydrogel was injected into the injury site and gained higher Basso, Beattie, and Bresnahan (BBB) scores which suggested that this new cellfree therapeutic approach is conducive to sensory and motor function recovery of SCI [110]. Peripheral nerve injury following traumatic accidents or surgical complications is a severe clinical problem resulting in sensory disturbances, paralysis, and locomotive disability. Because dental MSCs present a great privilege in neurogenic differentiation, they are a hopeful cell source to treat injured peripheral nerves, like the sciatic nerve and SLN [111-113]. For example, the sciatic nerve could be regenerated and repaired after hDPSC implantation or exosome derived from GMSC transplantation in a rat model with sciatic nerve defects $[112,113]$. Besides, Tsuruta et al. established a novel animal model of SLN injury, which was characterized as having weight loss and drinking behavior changes. The therapeutic effects of systemic administration of SHED-CM in this model showed functional recovery of the SLN and axonal regeneration [114]. Furthermore, hDPSCs have been suggested as an appropriate stem cell source for stroke treatment and acute cerebral ischemia $[115,116]$.

Also, dental MSCs play an important role in bone and cartilage tissue engineering. Campos et al. treated noncritical defects in an ovine model with the biomaterial Bonelike and hDPSCs, and obtained significant radiographic and microscopical evidence of improved bone regeneration [117]. hDPSCs also have been used to treat full-thickness articular cartilage defects. hDPSCs and PRP scaffolds were transplanted into full-thickness cartilage defects in rabbits, resulting in a significant improvement of impaired cartilage and formation of articular cartilage with hyaline-like and fibrocartilaginous tissue [118].

Furthermore, dental MSCs might be another choice for systemic lupus erythmatosus (SLE) therapy and are also effective in reducing a kidney glomerular lesion and perivascular inflammation infiltration [119]. And dental MSCs are able to treat diabetes by obtaining insulin-producing cells or improving diabetic polyneuropathy $[119,120]$.

\section{Conclusion}

Dental MSCs have been a precious stem cell source in regenerative medicine and have a great therapeutic application potential not only in oral diseases but also in various extraoral diseases. Here, a lot of evidence has demonstrated that dental MSCs are capable of multi-lineage differentiation and are conducive for regenerating and repairing dental tissue. Moreover, some clinical trials with dental MSCs have been completed and demonstrated the efficacy and safety of dental MSC-based therapy for oral diseases. However, these studies are limited, with a limited number of patients and a 
rather short-term follow-up, so more clinical trials are required before they can be applied effectively and safely in clinic. In addition to a significant potential in dental medicine, dental MSCs have already been considered as an alternative source for nerve and bone regeneration and have therapeutic potential for treating various diseases, such as neural impairment, stoke, bone and cartilage defects, SLE, and diabetes. However, thoroughly understanding the regulatory mechanism of dental MSCs is required before their wide application in clinic.

\section{Conflicts of Interest}

The authors declare that there are no conflicts of interest regarding the publication of this paper.

\section{Authors' Contributions}

Lu Gan and Ying Liu contributed equally to this work.

\section{Acknowledgments}

This work was supported by National Natural Science Foundation of China (NSFC) Grant 81800929 and Sichuan Science and Technology Program 2019JDRC0096 to Mian Wan; National Natural Science Foundation of China (NSFC) Grant 81771033 and Clinical Research Project/Foundation of West China Hospital of Stomatology Grant LCYJ2019-24 to Liwei Zheng; and Preeminent Youth Fund of Sichuan Province Grant 2016JQ0054 to Liwei Zheng.

\section{References}

[1] M. F. Pittenger, A. M. Mackay, S. C. Beck et al., "Multilineage potential of adult human mesenchymal stem cells," Science, vol. 284, no. 5411, pp. 143-147, 1999.

[2] F. Gao, S. M. Chiu, D. A. L. Motan et al., "Mesenchymal stem cells and immunomodulation: current status and future prospects," Cell Death \& Disease, vol. 7, no. 1, article e2062, 2016.

[3] R. Berebichez-Fridman and P. R. Montero-Olvera, "Sources and clinical applications of mesenchymal stem cells: stateof-the-art review," Sultan Qaboos University Medical Journal, vol. 18, no. 3, pp. 264-e277, 2018.

[4] Y. Han, X. Li, Y. Zhang, Y. Han, F. Chang, and J. Ding, "Mesenchymal stem cells for regenerative medicine," Cell, vol. 8, no. 8, p. 886, 2019.

[5] G. T.-J. Huang, S. Gronthos, and S. Shi, "Mesenchymal stem cells derived from dental tissues vs. those from other sources: their biology and role in regenerative medicine," Journal of Dental Research, vol. 88, no. 9, pp. 792-806, 2009.

[6] P. T. Sharpe, "Dental mesenchymal stem cells," Development, vol. 143, no. 13, pp. 2273-2280, 2016.

[7] E. P. Chalisserry, S. Y. Nam, S. H. Park, and S. Anil, "Therapeutic potential of dental stem cells," Journal of Tissue Engineering, vol. 8, 2016.

[8] G. Varga and G. Gerber, "Mesenchymal stem cells of dental origin as promising tools for neuroregeneration," Stem Cell Research \& Therapy, vol. 5, no. 2, p. 61, 2014.

[9] R. Patil, B. M. Kumar, W.-J. Lee et al., "Multilineage potential and proteomic profiling of human dental stem cells derived from a single donor," Experimental Cell Research, vol. 320, no. 1, pp. 92-107, 2014.

[10] J. Fujiyoshi, H. Yamaza, S. Sonoda et al., "Therapeutic potential of hepatocyte-like-cells converted from stem cells from human exfoliated deciduous teeth in fulminant Wilson's disease," Scientific Reports, vol. 9, no. 1, article 1535, 2019.

[11] O. Trubiani, J. Pizzicannella, S. Caputi et al., "Periodontal ligament stem cells: current knowledge and future perspectives," Stem Cells and Development, vol. 28, no. 15, pp. 9951003, 2019.

[12] K. Iohara, L. Zheng, H. Wake et al., "A novel stem cell source for vasculogenesis in ischemia: subfraction of side population cells from dental pulp," Stem Cells, vol. 26, no. 9, pp. 24082418, 2008.

[13] J. Liu, F. Yu, Y. Sun et al., "Concise reviews: characteristics and potential applications of human dental tissue-derived mesenchymal stem cells," Stem Cells, vol. 33, no. 3, pp. 627638, 2015.

[14] G. Orsini, P. Pagella, and T. A. Mitsiadis, "Modern trends in dental medicine: an update for internists," The American Journal of Medicine, vol. 131, no. 12, pp. 1425-1430, 2018.

[15] Q. Zhai, Z. Dong, W. Wang, B. Li, and Y. Jin, "Dental stem cell and dental tissue regeneration," Frontiers of Medicine, vol. 13, no. 2, pp. 152-159, 2019.

[16] M. Tatullo, B. Codispoti, F. Paduano, M. Nuzzolese, and I. Makeeva, "Strategic tools in regenerative and translational dentistry," International Journal of Molecular Sciences, vol. 20, no. 8, article 1879, 2019.

[17] P. Stanko, U. Altanerova, J. Jakubechova, V. Repiska, and C. Altaner, "Dental mesenchymal stem/stromal cells and their exosomes," Stem Cells International, vol. 2018, Article ID 8973613, 8 pages, 2018.

[18] Y. Yamada, S. Nakamura-Yamada, K. Kusano, and S. Baba, "Clinical potential and current progress of dental pulp stem cells for various systemic diseases in regenerative medicine: a concise review," International Journal of Molecular Sciences, vol. 20, no. 5, article 1132, 2019.

[19] S. Gronthos, M. Mankani, J. Brahim, P. G. Robey, and S. Shi, "Postnatal human dental pulp stem cells (DPSCs) in vitro and in vivo," Proceedings of the National Academy of Sciences of the United States of America, vol. 97, no. 25, pp. 1362513630, 2000.

[20] M. Miura, S. Gronthos, M. Zhao et al., "SHED: stem cells from human exfoliated deciduous teeth," Proceedings of the National Academy of Sciences of the United States of America, vol. 100, no. 10, pp. 5807-5812, 2003.

[21] W. Sonoyama, Y. Liu, D. Fang et al., "Mesenchymal stem cellmediated functional tooth regeneration in swine," PLoS One, vol. 1, no. 1, article e79, 2006.

[22] B.-M. Seo, M. Miura, S. Gronthos et al., "Investigation of multipotent postnatal stem cells from human periodontal ligament," The Lancet, vol. 364, no. 9429, pp. 149-155, 2004.

[23] Q. Zhang, S. Shi, Y. Liu et al., "Mesenchymal stem cells derived from human gingiva are capable of immunomodulatory functions and ameliorate inflammation-related tissue destruction in experimental colitis," Journal of Immunology, vol. 183, no. 12, pp. 7787-7798, 2009.

[24] C. Morsczeck, W. Götz, J. Schierholz et al., "Isolation of precursor cells (PCs) from human dental follicle of wisdom teeth," Matrix Biology, vol. 24, no. 2, pp. 155-165, 2005. 
[25] C. Han, Z. Yang, W. Zhou et al., "Periapical follicle stem cell: a promising candidate for cementum/periodontal ligament regeneration and bio-root engineering," Stem Cells and Development, vol. 19, no. 9, pp. 1405-1415, 2010.

[26] E. Ikeda, K. Yagi, M. Kojima et al., "Multipotent cells from the human third molar: feasibility of cell-based therapy for liver disease," Differentiation, vol. 76, no. 5, pp. 495-505, 2008.

[27] T. Matsubara, K. Suardita, M. Ishii et al., "Alveolar bone marrow as a cell source for regenerative medicine: differences between alveolar and iliac bone marrow stromal cells," Journal of Bone and Mineral Research, vol. 20, no. 3, pp. 399-409, 2005.

[28] T. Zhou, J. Pan, P. Wu et al., "Dental follicle cells: roles in development and beyond," Stem Cells International, vol. 2019, Article ID 9159605, 17 pages, 2019.

[29] I. Mortada and R. Mortada, "Dental pulp stem cells and osteogenesis: an update," Cytotechnology, vol. 70, no. 5, pp. 14791486, 2018.

[30] E. Anitua, M. Troya, and M. Zalduendo, "Progress in the use of dental pulp stem cells in regenerative medicine," Cytotechnology, vol. 20, no. 4, pp. 479-498, 2018.

[31] S. Sohn, Y. Park, S. Srikanth et al., "The role of ORAI1 in the odontogenic differentiation of human dental pulp stem cells," Journal of Dental Research, vol. 94, no. 11, pp. 1560-1567, 2015.

[32] G. S. da Silva, M. S. Moreira, K. A. Fukushima et al., "Current evidence of tissue engineering for dentine regeneration in animal models: a systematic review," Regenerative Medicine, vol. 15, no. 2, pp. 1345-1360, 2020.

[33] S. Y. Jeong, S. Lee, W. H. Choi, J. H. Jee, H.-R. Kim, and J. Yoo, "Fabrication of dentin-pulp-like organoids using dental-pulp stem cells," Cell, vol. 9, no. 3, p. 642, 2020.

[34] P. Pagella, S. Miran, E. Neto, I. Martin, M. Lamghari, and T. A. Mitsiadis, "Human dental pulp stem cells exhibit enhanced properties in comparison to human bone marrow stem cells on neurites outgrowth," The FASEB Journal, vol. 34, no. 4, pp. 5499-5511, 2020.

[35] Y. Kawase-Koga, Y. Fujii, D. Yamakawa, M. Sato, and D. Chikazu, "Identification of neurospheres generated from human dental pulp stem cells in xeno-/serum-free conditions," Regenerative Therapy, vol. 14, pp. 128-135, 2020.

[36] J. Jung, J.-W. Kim, H.-J. Moon, J. Y. Hong, and J. K. Hyun, "Characterization of neurogenic potential of dental pulp stem cells cultured in xeno/serum-free condition: in vitro and in vivo assessment," Stem Cells International, vol. 2016, Article ID 6921097, 12 pages, 2016.

[37] M. Nakashima, K. Iohara, M. Murakami et al., "Pulp regeneration by transplantation of dental pulp stem cells in pulpitis: a pilot clinical study," Stem Cell Research \& Therapy, vol. 8, no. 1, p. 61, 2017.

[38] P. Hilkens, A. Bronckaers, J. Ratajczak, P. Gervois, E. Wolfs, and I. Lambrichts, "The angiogenic potential of DPSCs and SCAPs in an in vivo model of dental pulp regeneration," Stem Cells International, vol. 2017, Article ID 2582080, 14 pages, 2017.

[39] M. M. Cordeiro, Z. Dong, T. Kaneko et al., "Dental pulp tissue engineering with stem cells from exfoliated deciduous teeth," Journal of Endodontics, vol. 34, no. 8, pp. 962-969, 2008.

[40] V. Rosa, Z. Zhang, R. H. M. Grande, and J. E. Nör, "Dental pulp tissue engineering in full-length human root canals," Journal of Dental Research, vol. 92, no. 11, pp. 970-975, 2013.
[41] J. Wang, X. Wang, Z. Sun et al., "Stem cells from humanexfoliated deciduous teeth can differentiate into dopaminergic neuron-like cells," Stem Cells and Development, vol. 19, no. 9, pp. 1375-1383, 2010.

[42] F. Nicola, M. R. Marques, F. Odorcyk et al., "Stem cells from human exfoliated deciduous teeth modulate early astrocyte response after spinal cord contusion," Molecular Neurobiology, vol. 56, no. 1, pp. 748-760, 2019.

[43] Y. Sugimura-Wakayama, W. Katagiri, M. Osugi et al., "Peripheral nerve regeneration by secretomes of stem cells from human exfoliated deciduous teeth," Stem Cells and Development, vol. 24, no. 22, pp. 2687-2699, 2015.

[44] K. Nakajima, R. Kunimatsu, K. Ando et al., "Comparison of the bone regeneration ability between stem cells from human exfoliated deciduous teeth, human dental pulp stem cells and human bone marrow mesenchymal stem cells," Biochemical and Biophysical Research Communications, vol. 497, no. 3, pp. 876-882, 2018.

[45] K. H. Kwack, J. M. Lee, S. H. Park, and H. W. Lee, "Human dental pulp stem cells suppress alloantigen-induced immunity by stimulating $\mathrm{T}$ cells to release transforming growth factor beta," Journal of Endodontics, vol. 43, no. 1, pp. 100-108, 2017.

[46] Y.-Y. Dai, S.-Y. Ni, K. Ma, Y.-S. Ma, Z.-S. Wang, and X.L. Zhao, "Stem cells from human exfoliated deciduous teeth correct the immune imbalance of allergic rhinitis via Treg cells in vivo and in vitro," Stem Cell Research \& Therapy, vol. 10, no. 1, p. 39, 2019.

[47] X. Gao, Z. Shen, M. Guan et al., "Immunomodulatory role of stem cells from human exfoliated deciduous teeth on periodontal regeneration," Tissue Engineering Part A, vol. 24, no. 17-18, pp. 1341-1353, 2018.

[48] W. Sonoyama, Y. Liu, T. Yamaza et al., "Characterization of the apical papilla and its residing stem cells from human immature permanent teeth: a pilot study," Journal of Endodontics, vol. 34, no. 2, pp. 166-171, 2008.

[49] C. Pelissari, A. F. C. Paris, A. Mantesso, and M. Trierveiler, "Apical papilla cells are capable of forming a pulplike tissue with odontoblastlike cells without the use of exogenous growth factors," Journal of Endodontics, vol. 44, no. 11, pp. 1671-1676, 2018.

[50] K. Chen, H. Xiong, Y. Huang, and C. Liu, "Comparative analysis of in vitro periodontal characteristics of stem cells from apical papilla (SCAP) and periodontal ligament stem cells (PDLSCs)," Archives of Oral Biology, vol. 58, no. 8, pp. 997-1006, 2013.

[51] P. De Berdt, J. Vanacker, B. Ucakar et al., "Dental apical papilla as therapy for spinal cord injury," Journal of Dental Research, vol. 94, no. 11, pp. 1575-1581, 2015.

[52] G. Ding, Y. Liu, Y. An et al., "Suppression of T cell proliferation by root apical papilla stem cells in vitro," Cells, Tissues, Organs, vol. 191, no. 5, pp. 357-364, 2010.

[53] C. A. G. McCulloch and A. H. Melcher, "Cell density and cell generation in the periodontal ligament of mice," The American Journal of Anatomy, vol. 167, no. 1, pp. 43-58, 1983.

[54] T. Iwata, M. Yamato, Z. Zhang et al., "Validation of human periodontal ligament-derived cells as a reliable source for cytotherapeutic use," Journal of Clinical Periodontology, vol. 37, no. 12, pp. 1088-1099, 2010.

[55] O. Trubiani, S. Guarnieri, F. Diomede et al., "Nuclear translocation of PKC $\alpha$ isoenzyme is involved in neurogenic 
commitment of human neural crest-derived periodontal ligament stem cells," Cellular Signalling, vol. 28, no. 11, pp. 16311641, 2016.

[56] G. Ding, Y. Liu, W. Wang et al., "Allogeneic periodontal ligament stem cell therapy for periodontitis in swine," Stem Cells, vol. 28, no. 10, pp. 1829-1838, 2010.

[57] F. Diomede, A. Gugliandolo, P. Cardelli et al., "Three-dimensional printed PLA scaffold and human gingival stem cellderived extracellular vesicles: a new tool for bone defect repair," Stem Cell Research \& Therapy, vol. 9, no. 1, p. 104, 2018.

[58] J. Li, S.-q. Xu, K. Zhang et al., "Treatment of gingival defects with gingival mesenchymal stem cells derived from human fetal gingival tissue in a rat model," Stem Cell Research \& Therapy, vol. 9, no. 1, p. 27, 2018.

[59] Q. Zhang, P. D. Nguyen, S. Shi, J. C. Burrell, D. K. Cullen, and A. D. le, "3D bio-printed scaffold-free nerve constructs with human gingiva-derived mesenchymal stem cells promote rat facial nerve regeneration," Scientific Reports, vol. 8, no. 1, article 6634, 2018.

[60] K. M. Fawzy El-Sayed and C. E. Dörfer, "Gingival mesenchymal stem/progenitor cells: a unique tissue engineering gem," Stem Cells International, vol. 2016, Article ID 7154327, 16 pages, 2016.

[61] Q. Zhang, A. L. Nguyen, S. Shi et al., "Three-dimensional spheroid culture of human gingiva-derived mesenchymal stem cells enhances mitigation of chemotherapy-induced oral mucositis," Stem Cells and Development, vol. 21, no. 6, pp. 937-947, 2012.

[62] S. Yildirim, N. Zibandeh, D. Genc, E. M. Ozcan, K. Goker, and T. Akkoc, "The comparison of the immunologic properties of stem cells isolated from human exfoliated deciduous teeth, dental pulp, and dental follicles," Stem Cells International, vol. 2016, Article ID 4682875, 15 pages, 2016.

[63] Y. Tian, D. Bai, W. Guo et al., "Comparison of human dental follicle cells and human periodontal ligament cells for dentin tissue regeneration," Regenerative Medicine, vol. 10, no. 4, pp. 461-479, 2015.

[64] S. Tomic, J. Djokic, S. Vasilijic et al., "Immunomodulatory properties of mesenchymal stem cells derived from dental pulp and dental follicle are susceptible to activation by tolllike receptor agonists," Stem Cells and Development, vol. 20, no. 4, pp. 695-708, 2011.

[65] P. N. Taşlı, S. Aydın, M. E. Yalvaç, and F. Şahin, "Bmp 2 and bmp 7 induce odonto- and osteogenesis of human tooth germ stem cells," Applied Biochemistry and Biotechnology, vol. 172, no. 6, pp. 3016-3025, 2014.

[66] A. C. Calikoglu Koyuncu, G. Gurel Pekozer, M. Ramazanoglu, G. Torun Kose, and V. Hasirci, "Cartilage tissue engineering on macroporous scaffolds using human tooth germ stem cells," Journal of Tissue Engineering and Regenerative Medicine, vol. 11, no. 3, pp. 765-777, 2017.

[67] X. Wang, H. Xing, G. Zhang et al., "Restoration of a Critical Mandibular Bone Defect Using Human Alveolar BoneDerived Stem Cells and Porous Nano-HA/Collagen/PLA Scaffold," Stem Cells International, vol. 2016, Article ID 8741641, 13 pages, 2016.

[68] T. Morotomi, A. Washio, and C. Kitamura, "Current and future options for dental pulp therapy," The Japanese Dental Science Review, vol. 55, no. 1, pp. 5-11, 2019.
[69] Y. Zheng, X. Y. Wang, Y. M. Wang et al., "Dentin regeneration using deciduous pulp stem/progenitor cells," Journal of Dental Research, vol. 91, no. 7, pp. 676-682, 2012.

[70] H. L. B. Tran and V. N. Doan, "Human dental pulp stem cells cultured onto dentin derived scaffold can regenerate dentinlike tissue in vivo," Cell and Tissue Banking, vol. 16, no. 4, pp. 559-568, 2015.

[71] R. Kuang, Z. Zhang, X. Jin et al., "Nanofibrous spongy microspheres enhance odontogenic differentiation of human dental pulp stem cells," Advanced Healthcare Materials, vol. 4, no. 13, pp. 1993-2000, 2015.

[72] M. Zhang, F. Jiang, X. Zhang et al., "The effects of plateletderived growth factor-BB on human dental pulp stem cells mediated dentin-pulp complex regeneration," Stem Cells Translational Medicine, vol. 6, no. 12, pp. 2126-2134, 2017.

[73] G. T. J. Huang, "Pulp and dentin tissue engineering and regeneration: current progress," Regenerative Medicine, vol. 4, no. 5, pp. 697-707, 2009.

[74] J.-B. Souron, A. Petiet, F. Decup et al., "Pulp cell tracking by radionuclide imaging for dental tissue engineering," Tissue Engineering Part C: Methods, vol. 20, no. 3, pp. 188-197, 2014.

[75] W. Jia, Y. Zhao, J. Yang et al., "Simvastatin Promotes Dental Pulp Stem Cell-induced Coronal Pulp Regeneration in Pulpotomized Teeth," Journal of Endodontics, vol. 42, no. 7, pp. 1049-1054, 2016.

[76] F. Mangione, M. EzEldeen, C. Bardet et al., "Implanted dental pulp cells fail to induce regeneration in partial pulpotomies," Journal of Dental Research, vol. 96, no. 12, pp. 1406-1413, 2017.

[77] M. Nakashima and K. Iohara, "Mobilized dental pulp stem cells for pulp regeneration: initiation of clinical trial," Journal of Endodontics, vol. 40, no. 4, pp. S26-S32, 2014.

[78] Y. Itoh, J. I. Sasaki, M. Hashimoto, C. Katata, M. Hayashi, and S. Imazato, "Pulp regeneration by 3-dimensional dental pulp stem cell constructs," Journal of Dental Research, vol. 97, no. 10, pp. 1137-1143, 2018.

[79] K. Xuan, B. Li, H. Guo et al., "Deciduous autologous tooth stem cells regenerate dental pulp after implantation into injured teeth," Science Translational Medicine, vol. 10, no. 455, article eaaf3227, 2018.

[80] G. Meza, D. Urrejola, N. Saint Jean et al., "Personalized cell therapy for pulpitis using autologous dental pulp stem cells and leukocyte platelet-rich fibrin: a case report," Journal of Endodontics, vol. 45, no. 2, pp. 144-149, 2019.

[81] D. F. Kinane, P. G. Stathopoulou, and P. N. Papapanou, "Periodontal diseases," Nature Reviews Disease Primers, vol. 3, no. 1, article 17038, 2017.

[82] T. Ouchi and T. Nakagawa, "Mesenchymal stem cell-based tissue regeneration therapies for periodontitis," Regenerative Therapy, vol. 14, pp. 72-78, 2020.

[83] J. Nuñez, F. Vignoletti, R. G. Caffesse, and M. Sanz, "Cellular therapy in periodontal regeneration," Periodontology 2000, vol. 79, no. 1, pp. 107-116, 2019.

[84] G. Li, N. Han, X. Zhang et al., "Local injection of allogeneic stem cells from apical papilla enhanced periodontal tissue regeneration in minipig model of periodontitis," BioMed Research International, vol. 2018, Article ID 3960798, 8 pages, 2018. 
[85] G. Li, N. Han, H. Yang et al., "SFRP2 promotes stem cells from apical papilla-mediated periodontal tissue regeneration in miniature pig," Journal of Oral Rehabilitation, 2019.

[86] K. Iwasaki, K. Akazawa, M. Nagata et al., "The fate of transplanted periodontal ligament stem cells in surgically created periodontal defects in rats," International Journal of Molecular Sciences, vol. 20, no. 1, p. 192, 2019.

[87] J. Qiu, X. Wang, H. Zhou et al., "Enhancement of periodontal tissue regeneration by conditioned media from gingivaderived or periodontal ligament-derived mesenchymal stem cells: a comparative study in rats," Stem Cell Research \& Therapy, vol. 11, no. 1, p. 42, 2020.

[88] F. Feng, K. Akiyama, Y. Liu et al., "Utility of PDL progenitors for in vivo tissue regeneration: a report of 3 cases," Oral Diseases, vol. 16, no. 1, pp. 20-28, 2010.

[89] K. L. Vandana, H. Ryana, and P. J. Dalvi, “Autologous periodontal stem cell assistance in periodontal regeneration technique (SAI-PRT) in the treatment of periodontal intrabony defects: a case report with one-year follow-up," Journal of Dental Research, Dental Clinics, Dental Prospects, vol. 11, no. 2, pp. 123-126, 2017.

[90] M. Aimetti, F. Ferrarotti, M. Gamba, M. Giraudi, and F. Romano, "Regenerative treatment of periodontal intrabony defects using autologous dental pulp stem cells: a 1year follow-up case series," The International Journal of Periodontics \& Restorative Dentistry, vol. 38, no. 1, pp. 51-58, 2018.

[91] F. Ferrarotti, F. Romano, M. N. Gamba et al., "Human intrabony defect regeneration with micrografts containing dental pulp stem cells: a randomized controlled clinical trial," Journal of Clinical Periodontology, vol. 45, no. 7, pp. 841-850, 2018.

[92] B. Hernández-Monjaraz, E. Santiago-Osorio, E. LedesmaMartínez, A. Alcauter-Zavala, and V. M. Mendoza-Núñez, "Retrieval of a periodontally compromised tooth by allogeneic grafting of mesenchymal stem cells from dental pulp: a case report," The Journal of International Medical Research, vol. 46, no. 7, pp. 2983-2993, 2018.

[93] D. Hughes and B. Song, "Dental and nondental stem cell based regeneration of the craniofacial region: a tissue based approach," Stem Cells International, vol. 2016, Article ID 8307195, 20 pages, 2016.

[94] X. Zhu, J. Liu, Z. Yu et al., “A miniature swine model for stem cell-based de novo regeneration of dental pulp and dentinlike tissue," Tissue Engineering Part C: Methods, vol. 24, no. 2, pp. 108-120, 2018.

[95] F. Wei, T. Song, G. Ding et al., "Functional tooth restoration by allogeneic mesenchymal stem cell-based bio-root regeneration in swine," Stem Cells and Development, vol. 22, no. 12, pp. 1752-1762, 2013.

[96] M. M. Hasani-Sadrabadi, P. Sarrion, S. Pouraghaei et al., “An engineered cell-laden adhesive hydrogel promotes craniofacial bone tissue regeneration in rats," Science Translational Medicine, vol. 12, no. 534, article eaay6853, 2020.

[97] H. Bakhtiar, A. Mazidi S, S. Mohammadi Asl et al., “The role of stem cell therapy in regeneration of dentine-pulp complex: a systematic review," Progress in Biomaterials, vol. 7, no. 4, pp. 249-268, 2018.

[98] L. Hu, Y. Liu, and S. Wang, "Stem cell-based tooth and periodontal regeneration," Oral Diseases, vol. 24, no. 5, pp. 696705, 2018.
[99] Y. Tsumanuma, T. Iwata, K. Washio et al., "Comparison of different tissue-derived stem cell sheets for periodontal regeneration in a canine 1-wall defect model," Biomaterials, vol. 32, no. 25, pp. 5819-5825, 2011.

[100] S. Eramo, A. Natali, R. Pinna, and E. Milia, "Dental pulp regeneration via cell homing," International Endodontic Journal, vol. 51, no. 4, pp. 405-419, 2018.

[101] S. El Moshy, I. A. Radwan, D. Rady et al., "Dental stem cellderived secretome/conditioned medium: the future for regenerative therapeutic applications," Stem Cells International, vol. 2020, Article ID 7593402, 29 pages, 2020.

[102] M. Nagata, K. Iwasaki, K. Akazawa et al., "Conditioned medium from periodontal ligament stem cells enhances periodontal regeneration," Tissue Engineering Part A, vol. 23, no. 9-10, pp. 367-377, 2017.

[103] R. T. Stuepp, P. B. Delben, F. Modolo, A. G. Trentin, R. C. Garcez, and M. T. Biz, "Human dental pulp stem cells in rat mandibular bone defects," Cells, Tissues, Organs, vol. 207, no. 3-4, pp. 138-148, 2020.

[104] D. M. Saez, R. T. Sasaki, D. d. O. Martins, M. Chacur, I. Kerkis, and M. C. P. da Silva, "Rat facial nerve regeneration with human immature dental pulp stem cells," Cell Transplantation, vol. 28, no. 12, pp. 1573-1584, 2019.

[105] S. J. Cui, T. Zhang, Y. Fu et al., "DPSCs attenuate experimental progressive TMJ arthritis by inhibiting the STAT1 pathway," Journal of Dental Research, vol. 99, no. 4, pp. 446-455, 2020.

[106] Y. Zhang, S. Shi, Q. Xu, Q. Zhang, R. M. Shanti, and A. D. Le, "SIS-ECM laden with GMSC-derived exosomes promote taste bud regeneration," Journal of Dental Research, vol. 98, no. 2, pp. 225-233, 2019.

[107] D. Wang, Y. Wang, W. Tian, and J. Pan, "Advances of tooth-derived stem cells in neural diseases treatments and nerve tissue regeneration," Cell Proliferation, vol. 52, no. 3, article e12572, 2019.

[108] Y. Xu, M. Chen, T. Zhang et al., "Spinal cord regeneration using dental stem cell-based therapies," Acta Neurobiologiae Experimentalis, vol. 79, no. 4, pp. 319-327, 2019.

[109] S. Kabatas, C. S. Demir, E. Civelek et al., "Neuronal regeneration in injured rat spinal cord after human dental pulp derived neural crest stem cell transplantation," Bratislava Medical Journal, vol. 119, no. 3, pp. 143-151, 2018.

[110] R. Asadi-Golshan, V. Razban, E. Mirzaei et al., "Sensory and motor behavior evidences supporting the usefulness of conditioned medium from dental pulp-derived stem cells in spinal cord injury in rats," Asian Spine Journal, vol. 12, no. 5, pp. 785-793, 2018.

[111] C. Prado, P. Fratini, G. de Sá Schiavo Matias et al., "Combination of stem cells from deciduous teeth and electroacupuncture for therapy in dogs with chronic spinal cord injury: a pilot study," Research in Veterinary Science, vol. 123, pp. 247-251, 2019.

[112] Y. Okuwa, T. Toriumi, H. Nakayama et al., "Transplantation effects of dental pulp-derived cells on peripheral nerve regeneration in crushed sciatic nerve injury," Journal of Oral Science, vol. 60, no. 4, pp. 526-535, 2018.

[113] F. Rao, D. Zhang, T. Fang et al., "Exosomes from Human Gingiva-Derived Mesenchymal Stem Cells Combined with Biodegradable Chitin Conduits Promote Rat Sciatic Nerve Regeneration," Stem Cells International, vol. 2019, Article ID 2546367, 12 pages, 2019. 
[114] T. Tsuruta, K. Sakai, J. Watanabe, W. Katagiri, and H. Hibi, "Dental pulp-derived stem cell conditioned medium to regenerate peripheral nerves in a novel animal model of dysphagia," PLoS One, vol. 13, no. 12, article e0208938, 2018.

[115] M. R. Gancheva, K. L. Kremer, S. Gronthos, and S. A. Koblar, "Using dental pulp stem cells for stroke therapy," Frontiers in Neurology, vol. 10, p. 422, 2019.

[116] C. Nito, K. Sowa, M. Nakajima et al., "Transplantation of human dental pulp stem cells ameliorates brain damage following acute cerebral ischemia," Biomedicine \& Pharmacotherapy, vol. 108, pp. 1005-1014, 2018.

[117] J. M. Campos, A. C. Sousa, A. R. Caseiro et al., "Dental pulp stem cells and Bonelike ${ }^{\circledR}$ for bone regeneration in ovine model," Regenerative Biomaterials, vol. 6, no. 1, pp. 49-59, 2019.

[118] X. Tang, W. Li, X. Wen et al., "Transplantation of dental tissue-derived mesenchymal stem cells ameliorates nephritis in lupus mice," Annals of Translational Medicine, vol. 7, no. 7, p. 132, 2019.

[119] B. Xu, D. Fan, Y. Zhao et al., "Three-dimensional culture promotes the differentiation of human dental pulp mesenchymal stem cells into insulin-producing cells for improving the diabetes therapy," Frontiers in Pharmacology, vol. 10, article 1576, 2020.

[120] E. Miura-Yura, S. Tsunekawa, K. Naruse et al., "Secreted factors from cultured dental pulp stem cells promoted neurite outgrowth of dorsal root ganglion neurons and ameliorated neural functions in streptozotocin-induced diabetic mice," Journal of Diabetes Investigation, vol. 11, no. 1, pp. 28-38, 2020. 